American Journal of Qualitative Research

June 2021, Vol. 5 No. 1, pp. 85-93

https://doi.org/10.29333/ajqr/10813

(C) 2021 AJQR. http://www.ajqr.org
AMERICAN

JOURNALOFQUALTAIUE

RESEARCH

ISSN: 2576-2141

\title{
Use of Video Conferencing to Facilitate Focus Groups for Qualitative Data Collection
}

\author{
Scott B. Greenspan ${ }^{1}$, Kelsey L. Gordon, Sara A. Whitcomb, and Alexandra A. Lauterbach \\ University of Massachusetts Amherst, USA
}

\begin{abstract}
This paper explores the utility in collecting qualitative data by employing synchronous focus groups via web-based video conferencing software such as Zoom. The authors underscore benefits and drawbacks of this data collection modality by reviewing recent interdisciplinary literature. Authors also bring forth an example of using Zoom to facilitate synchronous focus groups for a recent research study. The study centered on school psychologists' perspectives of implementing physical activity within schools to support the mental health outcomes of youth and school communities. Authors offer reflections, lessons learned, and provide suggestions for employing successful online focus groups. Researchers that are interested in employing online focus groups should thoroughly assess if participants have access to and comfortability with technology. Such assessment data may provide insight into whether online focus groups serve as an appropriate modality. Further, Implications and suggestions for research are addressed.
\end{abstract}

KEYWORDS: Focus Groups, Grounded Theory, Qualitative Research, Research Methods, School Psychology, Video Conferencing.

Focus groups are a robust modality for data collection given their "explicit use of group interaction to produce data." (Stewart \& Williams, 2005, p. 396). Qualitative researchers have explored benefits and drawbacks to employing focus groups online. In the last two decades, researchers have discussed the utility in employing asynchronous and synchronous focus groups using email and other text-based formats (Kenny, 2005; Stewart \& William, 2005; Williams et al., 2012). With the increase in reliable audio-visual technology, newer literature highlights the promise in employing focus groups using online video conferencing software (Han et al., 2019; Tuttas, 2015; Kite \& Phongsavan, 2017).

\section{Online Synchronous Focus Groups via Web Conferencing}

In recent years, scholars have explored the benefits and drawbacks of employing synchronous focus groups through web conferencing software (e.g., Zoom, Adobe Connect). Focus group facilitators and participants can have real-time conversations and interact auditorily and visually. This is viewed as an authentic way to gather data (Tuttas, 2015; Janghorban et al., 2014), though it is imperative that researchers are planful as a measure to potentially off-set barriers

\footnotetext{
${ }^{1}$ Correspondent Author E-Mail: greenspan.sb@gmail.com
} 
(e.g., technical concerns, having an underrepresented sample; Chase \& Alvarez, 2000; Janghorban et al., 2014; Suarez-Balcazar et al., 2009).

Benefits of this data collection modality involves the feasibility and cost-effective nature of collecting data from participants located in various geographic locations, thus allowing for a greater dispersion of participants (Irani, 2019; Janghorban et al., 2014). Further, participants can engage in focus groups within convenient locations and limit travel (Janghorban et al., 2014). Additionally, scholars have discussed that synchronous focus groups mirror in-person conversations where participants can effectively converse with one person speaking at a time (Tuttas, 2015) and can bring forth similar high-quality data (Janghorban et al., 2014).

A notable drawback of this modality involves technical issues (e.g., sound cutting out, weak internet connection; Tuttas, 2015; Kite \& Phongsavan, 2017), and in response, scholars have worked on piloting focus groups (Tuttas, 2015) and providing tutorials to participants (Kite \& Phongsavan, 2017) to ameliorate these concerns. Further, this modality is most beneficial if participants have access to a fast internet connection and uphold competency in technology (Janghorban et al., 2014).

Past research has underscored that individuals from low-income communities, many of whom have minoritized racial and ethnic identities, experience barriers to engaging in research requiring internet access (Suarez-Balcazar et al., 2009). These barriers are due to inconsistent access to technology-centered education (Suarez-Balcazar et al., 2009). Current research suggests that most Americans own a smart phone (81\%), laptop/desktop (74\%), or tablet computer (52\%) (Pew Research Center, 2019). With this indicated, it is critical that researchers consider equity and access to ensure that diverse identities and perspectives are represented in research.

Further, given that participants engage in online focus groups within a convenient location such as their homes, they could become more distracted (e.g., children in the background; Tuttas, 2015). Subsequently, in the online context, researchers are unable to observe body language to gauge social cues as effectively as they would in an in-person context (Irani, 2019; Tuttas, 2015). Thus, if a researcher needs to observe non-verbal body language (e.g., body posture, where participants are focusing their gaze, who participants chose to sit next to) for their data collection purposes, an in-person focus group may be more appropriate than a focus group held within an online context. Irani (2019) suggests that when collecting highly sensitive information from participants, in-person data-collection may be more appropriate, as physical proximity may be more comforting for participants. Further, scholars have also underscored a significant attrition rate within this online modality (Kite \& Phongsavan, 2017; Tuttas, 2015). Tuttas (2015) urges researchers to anticipate an attrition rate of $50 \%$ or greater.

\section{Physical Activity and School-Based Mental Health Study}

This study explored school psychologists' $(N=20)$ perspectives of incorporating physical activity within mental health intervention service delivery in school settings. This study was conducted in Summer 2019 and was approved by the Institution Review Board at University of Massachusetts Amherst. The sample inclusion criteria involved school psychologists practicing within a public elementary school setting in the United States. There were 18 female participants and 2 male participants. All participants identified as White and Non-Hispanic. No participants identified as transgender, non-binary, or any other gender identity. Further, 12 participants were specialist level practitioners, and 8 participants were doctoral level practitioners. Participants were from the Midwestern (10\%), Northeastern (15\%), Southern (45\%), and Western (30\%) regions of the United States. 
There were robust online recruitment and screening processes to obtain an appropriate sample for the scope of the study. Specifically, participants were informed about the study through social media and professional listservs. Recruitment materials contained a link to a screener to determine eligibility status. Those eligible participants were then directed to a demographic questionnaire and a consent form. This information was then reviewed by the primary investigator (PI) to ensure consistency of responses, and in turn, eligibility. Following, eligible participants were sent a link to sign up for a focus group slot using an electronic signup sheet (signupgenius.com). They were sent a confirmation e-mail after signing up for the group and a reminder e-mail within 24-hours of the group start time. To prevent confusion, given the many time zones in the United States, the PI noted in most focus group related communication that the groups would be held in Eastern Standard Time (EST). A research incentive was utilized, as participants would receive a \$35 Amazon gift card upon completion of the focus group.

There were eight total sessions, including one session with one participant, four sessions with two participants, one session with three participants, and two sessions with four participants. The inconsistency in the numbers of participants per session was largely due to non-attendance or scheduling concerns among participants. The researchers strived to obtain focus groups made up of five to six participants, in accordance with recent literature suggesting that focus groups should have a minimum number of five or six participants and a maximum number of seven to ten participants (Carey \& Asbury, 2012; Morgan, 1996). Each session was scheduled for 90-minutes, and both Author 1, the primary investigator (PI) and Author 2, a research collaborator (hereby referred to as research team) were present.

The PI facilitated the session using a semi-structured interview protocol, and the research collaborator took notes and supported participants with technical difficulties by using the private messaging feature in Zoom. Each focus group session was audio-recorded using Zoom audiorecord feature as well as a back-up audio recorder. The audio obtained from the focus group recordings were seamlessly sent to professional transcribers through Rev.com. Following the completion of transcription, the research collaborator checked each transcript for accuracy in accordance with the in-session notes and audio recordings.

The research team utilized a grounded theory approach (Corbin \& Strauss, 2015). Sociologists Glaser and Strauss (1967) highlight that grounded theory uses the data to guide the development of a specific theory rather than "[using] theory generated by logical deduction from a priori assumptions" (Glaser \& Strauss, 1967, p. 3). Thus, in grounded theory, raw data form categories which through rigorous analysis illuminates an overarching theory.

All focus groups were transcribed and imported into NVivo 12 software for qualitative analysis. The research team analyzed the data in three unique stages, including open coding, axial coding, and selective coding (Corbin \& Strauss, 2015). Authors also used a constant comparative process (Corbin \& Strauss, 2015) to compare codes across transcript to support the amalgamation of rich themes. A coding book was formed throughout the process that served to document the name and definition of codes.

Open coding involves breaking down the data to effectively support sound interpretation (Corbin \& Strauss, 2015). The research team members read through the transcript and assigned code names while comparing the code labels to one another. They engaged in collaborative coding for the first two transcripts to develop familiarity with the process. They also received training from an expert in grounded theory to support our understanding of the coding process. When the research team members had sound consistency in the coding process, one member would code while the other member would check the coding. The research team met to reconcile differences and establish consensus for each transcript. 
In axial coding, codes are categorized and relationships within the data were explored (Corbin \& Strauss, 2015). The research team explored underlying meanings of the codes by assigning code-types to categories. In the third analytical component, selective coding, the research team explored the "core category" that unites the categories (Corbin \& Strauss, 2015). In this stage, the research team members were able to determine what components were essential to the facilitation of the theoretical model that was developed. The research team independently engaged in this axial coding process before coming together the review differences and develop consensus.

After the seventh transcript the codes were consistently repeating and new information was scant, suggesting that the data were nearing saturation (Corbin \& Strauss, 2015). Following coding of the eighth transcript, it was confirmed that data were saturated. Broadly, results of the study illuminated rich systemic facilitators and barriers to incorporating physical activity within schools to promote the mental health outcomes of youth and school communities.

\section{Reflections}

The aforementioned study provides important insights into using online synchronous focus groups via web conferencing. In alignment with the literature, the PI was able to recruit and conduct focus group interviews with a geographically diverse sample of participants (Irani, 2019; Janghorban et al., 2014; Kenny, 2005). The 20 participants were from the Midwestern (10\%), Northeastern (15\%), Southern (45\%), and Western (30\%) regions of the United States. This allowed for a diversity of perspectives that might not otherwise be obtained in one geographic area. Factors that may have contributed to diversity in geographical residence may include the use of online recruitment and the flexibility of scheduling afforded by the virtual focus group setting.

Further, to reduce interruptions for technological issues (e.g., sound cutting out, weak internet connection), the PI facilitated the session while the research collaborator simultaneously provided participants with technical support and answered questions using the private messaging feature. Technical support challenges included trouble shooting audio, video, and connection issues and providing participants with the zoom meeting phone number as needed. The PI did not provide a tutorial of the web-conferencing software before the start of the group, which in retrospect may have been a helpful measure to off-set technical issues (Kite \& Phongsavan, 2017).

During the focus groups, the PI directed the participants to respond to questions in a "popcorn style," non-ordered manner which helped to ensure sessions remained in the 90-minute time frame. In line with existing literature, participants were able to have respectful back and forth dialogue and generally showed interest in one another (Tuttas, 2015). The PI made a concerted effort to ask quieter participants if they would like to provide further contribution to specific prompts which often sparked increased dialogue.

In line with existing literature, drawbacks that arose included issues with technology such as background noise interference, freezing of video, and occasional sound cutting out with participant and facilitator audio (Kite \& Phongsavan, 2017; Tuttas, 2015). This interrupted the flow of conversations and made it difficult for the facilitators and participants to hear and follow the conversations.

When technology challenges arose, participants were provided with a phone number to call into the session. On occasion, distractions (e.g., driving, using multiple devices, pets, or caring for children) would impede participants' ability to focus, which has also been documented in the literature (Tuttas, 2015). As such, beginning the focus group sessions with a clear set of expectations may have helped to reduce some of these challenges (e.g., encouraging participants to sit in a quiet space free of distractions, checking for internet connection stability, directing 
attention toward one device, etc.). Further, despite participants signing up for focus group time slots, there was at least one participant within each session who did not attend, which concords with literature highlighting the significant attrition rate in this data collection modality (Kite \& Phongsavan, 2017; Tuttas, 2015). The continuous "no-shows" led to a smaller sample size than originally planned which fortunately did not impact data saturation.

It is noteworthy that this sample was homogenous and lacked ethnic, racial, and gender diversity. All participants identified as White, Non-Hispanic, and cisgender. Further, 90\% of participants were female. This could be attributed to a multitude of factors including the underrepresentation of participants from diverse groups in online research (Suarez-Balcazar et al., 2009) as well as within the field of school psychology (e.g., Walcott \& Hyson, 2018). While the field has been focused on the inclusion of more diverse identities (Foroutani, 2020; Grapin et al., 2016; Nguyen et al. 2018; Walcott \& Hyson, 2018), demographic data collected approximately 6 years ago highlights that the field was $86.3 \%$ White, 5.5\% Black or African American, 2.9\% Asian, and $4.0 \%$ other or multiracial (Walcott \& Hyson, 2018). Further, based on this data, $83.7 \%$ of school psychologists identified as female, $16.2 \%$ identified as male, and $.1 \%$ identified as agender (Walcott \& Hyson, 2018).

It is likely that school psychologists have access to technology given the importance and necessity of using computers to conduct their work (e.g., writing reports, communicating with family and community stakeholders via e-mail). However, it would be important to formally assess if participants could access an online focus group. For example, if assessment data indicated that participants in a sample had inconsistent access to technology or did not have a strong understanding of using technology, researchers may consider partnering with community resources (e.g., libraries, community centers) to lend assistance as well as private and comfortable spaces for participants to access. It may also be the case that an in-person focus group would serve as a more appropriate modality, especially for participants who have difficulty accessing technology.

Of note, in the physical activity and school-based mental health study, it also appeared that participants had difficulty reading one another's body language to determine when it was most appropriate to speak up and contribute to the conversations. This at times led to periods of silence as well as participants speaking over one another. While scholars have discussed that it can be difficult for researchers to observe body language in the online context (Irani, 2019; Tuttas, 2015), this is a critical consideration given the interactional tone of focus groups (Stewart \& Williams, 2005).

There were occasions throughout each focus group in which one or two participants heavily dominated the discussion, while others responded less. In the future, it may be useful for the facilitator to encourage responding in a more structured and timed manner, giving each participant more equal airtime, or more prompted opportunities to speak.

Another barrier that arose included participants coming into the session after it started. This interrupted the focus group and the participant seemed notably embarrassed. In this instance, the PI asked that particular participant to reschedule for another focus group. A suggestion to avoid that challenge from arising would be to utilize a waiting room feature and a research team member could send a private message to the late arrival (while they are in the waiting room) and inform them that that the group has started, and they can reschedule. If the focus group was not fully underway, a research team member could provide the participant with an update and then admit them to the room upon ensuring that they understand the focus group processes. The waiting room feature would serve to prevent disruptions to the overall group and better moderate when participants are able to join. 


\section{Conclusion}

In light of the burgeoning literature base focused on synchronous online focus groups coupled with reflections from the physical activity and school-based mental health study, this is a promising modality to collect qualitative data. However, it is important that researchers consider a host of factors before determining if this is the most appropriate method.

Firstly, while online focus groups have the ability to collect rich data just as an in-person focus group (Janghorban et al., 2014), it is important to bear in mind that recruiting the appropriate sample is a critical precursor. Given diverse samples have been significantly underrepresented in internet research (Suarez-Balcazar et al., 2009), it would behoove researchers to assess prospective participants' access to technology to ensure that diverse perspectives are represented within the data. Such an assessment could utilize published data from trusted sources or asking prospective participants through questionnaires or conversation. This will provide the researcher with a more comprehensive understanding if an in-person group might be more appropriate than an online group. Such an assessment measure may serve as an important contribution to this field of research.

In line with Tuttas's (2015) suggestion, researchers should plan for a significant attrition rate when employing focus groups in the online context. Thus, it is recommended that researchers seek motivational incentives (e.g., gift cards) while also over-recruiting to obtain a sample that can provide adequate data. To this end, researchers should consider the most appropriate webconferencing format for the study (Tuttas, 2015). Participants within certain professional and school contexts may have more experience with certain software (e.g., Zoom, Adobe Connect), and thus, utilizing that particular software in the study could prevent technological concerns due to participant's familiarity. Researchers might also explore if their institutions have subscriptions for particular web-conferencing software to off-set cost.

Researchers are also encouraged to offer technical assistance to participants throughout the entire study process. This may take shape as providing participants with a tutorial beforehand (Kite \& Phongsavan, 2017) or even piloting focus groups to work out any potential technical issues that could arise (Tuttas, 2015). When planning for the successful implementation of focus groups, researchers should ask themselves "What information am I assuming participants know?" (e.g., muting the microphone when not speaking/ enabling the microphone prior to speaking, turning video on) and then explicitly state such information to participants. Recruiting a research collaborator or assistant to support individual participants with technology or other matters during the group (e.g., informing participants of the question or prompt if they missed it) is beneficial so that the group is not interrupted and can proceed as planned while participants are obtaining the support they need.

With increasing technological innovation, online synchronous focus groups are a promising modality to collect qualitative data. Researchers should take caution in determining if online focus groups are the most appropriate modality by thinking through participants' abilities to access the study and if the content of the study would be better suited in an in-person context (e.g., a study focused on sensitive topics [Irani 2019], recruiting a sample that has limited or inconsistent access to technology [Suarez-Balcazar et al., 2009]). Researchers that employ online synchronous focus groups are encouraged to detail its methodological facilitators and barriers in publications to inform future research. 


\section{Figure 1}

Suggestions for Researchers Using Video Conferencing to Facilitate Synchronous Focus Groups

\section{Increase Participants and Reduce Attrition}

Utilize online recruitment methods

Utilize online scheduling tools

Provide a wide range of flexibility in scheduling options for participants

Provide motivational incentives for focus group participation (e.g., gift cards)

\section{Ameliorating Technology Challenges}

Pilot focus groups

Conduct web-conferencing tutorials with participants

Provide a call-in number for focus group session

Enable a waiting room feature

\section{Focus Group Structure and Facilitation}

Designate a research collaborator/ research assistant to support with technological concerns

Set clear expectations for the session

Use a facilitation method that encourages an interactional tone

Encourage equitable opportunities for airtime between participations

Utilize chat feature to troubleshoot technology challenges

Utilize chat feature to manage participants' questions

Ensure participants have privacy and can effectively engage in the focus group

\section{Considerations}

Consider issues of equity and access to technology

Consider participant diversity and possibilities/limitations for online versus in-person modalities

Consider assessing if the sample of interest would have ease of access to video conferencing software

Consider if there is cost associated with web conferencing software and how that may impact access

Consider participant familiarity with the web-conferencing tool

Consider if partnering with community resources (e.g., libraries, community centers) may foster increased engagement in online research

Consider opportunities/limitations of online versus in person participant-participant interactions $\&$ facilitator-participant interactions

Note: Refer to Reflections and Conclusions sections of this manuscript for detailed explanations of these suggestions.

\section{Acknowledgements}

The physical activity and school-based mental health study that was discussed in this paper was supported by The Mary Margaret Whitaker Webster Award, College of Education, University of Massachusetts Amherst and The McCormick Doctoral Dissertation Grant, College of Education, University of Massachusetts Amherst 


\section{References}

Carey, M. A., \& Asbury, J. (2012). Focus group research. Left Coast Press, Inc.

Chase, L., \& Alvarez, J. (2000). Internet research: The role of the focus group. Library \& Information Science Research, 22(4), 357-369. http://doi.org/10.1016/S07408188(00)00050-5

Corbin, J., \& Strauss, A. L. (2015). Basics of qualitative research (4th ed.). Sage Publications.

Foroutani, Y. (2020). Ethnic or Religious Identities? Multicultural Analysis in Australia from Socio-Demographic Perspective. Journal of Ethnic and Cultural Studies, 7(1), 1-19.

Glaser, B., \& Strauss, A. (1967). The discovery of Grounded Theory: Strategies for qualitative research. Sociology Press.

Grapin, S. L., Bocanegra, J. O., Green, T. D., Lee, E. T., \& Jaafar, D. (2016). Increasing diversity in school psychology: Uniting the efforts of institutions, faculty, students, and practitioners. Contemporary School Psychology, 20(4), 345-355. http://doi.org/10.1007/s40688-0160092-z

Han, J., Torok, M., Gale, N., Wong, Q. J., Werner-Seidler, A., Hetrick, S. E., \& Christensen, H. (2019). Use of web conferencing technology for conducting online focus groups among young people with lived experience of suicidal thoughts: mixed methods research. JMIR mental health, 6(10), e14191. http://doi.org/10.2196/14191

Irani, E. (2019). The use of videoconferencing for qualitative interviewing: Opportunities, challenges, and considerations. Clinical Nursing Research, 28(1), 3-8. https://doi.org/10.1177/1054773818803170

Janghorban, R., Roudsari, R. L., \& Taghipour, A. (2014). Skype interviewing: The new generation of online synchronous interview in qualitative research. International Journal of Qualitative Studies on Health \& Well-Being, 9(1), 24152. http://doi.org/10.3402/qhw.v9.24152

Kenny, A. J. (2005). Interaction in cyberspace: An online focus group. Journal of Advanced Nursing, 49(4), 414-422. http://doi.org/10.1111/j.1365-2648.2004.03305.x

Kite, J., \& Phongsavan, P. (2017). Insights for conducting real-time focus groups online using a web conferencing service. Flo00Research, 1-12. http://doi.org/10.12688/f1000research.10427.1

Morgan, D. L. (1996). Focus groups as qualitative research. Sage publications.

Nguyen, P., Corona, R., DeCarlo, M. P., Yaros, A., Le, A. T., \& Compton, K. (2018). Help Seeking Behavior in a Diverse Sample of Asian American Adults. Journal of Ethnic and Cultural Studies, 5(2), 1-15.

Pew Research Center. (2019). Mobile fact sheet. https://www.pewresearch.org/internet/factsheet/mobile/

Stewart, K., \& Williams, M. (2005). Researching online populations: The use of online focus groups for social research. Qualitative Research, 5(4), 395-416. http://doi.org/10.1177/1468794105056916

Suarez-Balcazar, Y., Balcazar, F. E., \& Taylor-Ritzler, T. (2009). Using the internet to conduct research with culturally diverse populations: Challenges and opportunities. Cultural Diversity \& Ethnic Minority Psychology, 15(1), 96-104. http://doi.org/10.1037/a0013179

Tuttas, C. A. (2015). Lessons learned using web conference technology for online focus group interviews. Qualitative Health Research, 25(1), 122-133. http://doi.org/10.1177/1049732314549602 
Walcott, C. M., \& Hyson, D. (2018). Results from the NASP 2015 membership survey, part one: Demographics and employment conditions [Research report]. National Association of School Psychologists.

Williams, S., Clausen, M. G., Robertson, A., Peacock, S., \& McPherson, K. (2012). Methodological reflections on the use of asynchronous online focus groups in health research. International Journal of Qualitative Methods, 11(4), 368-383. http://doi.org/10.1177/160940691201100405

\section{Notes on Contributors}

Scott B. Greenspan, Ph.D., NCSP, is a recent graduate of the School Psychology PhD program at University of Massachusetts Amherst. He conducted his dissertation study on school psychologists' perspectives of incorporating physical activity within mental health services. Dr. Greenspan's research interests focus on evidence-based mental health interventions, physical activity, and affirming psychosocial supports for LGBTQ+ youth. He is currently a Postdoctoral Associate at Wediko Children's Services, The Home for Little Wanderers, in Boston, Massachusetts.

Kelsey L. Gordon, M.Ed., is a current graduate student of the School Psychology Ph.D. program at the University of Massachusetts Amherst. Her research interests focus on supporting youth navigating severe emotional and behavioral challenges and supporting culturallysustaining/trauma-informed school-based practices. She is currently a graduate research assistant with the Behavioral health Integrated Resources for Children Project of Massachusetts (BIRCh Project).

Sara A. Whitcomb, Ph.D., LP, is an Associate Professor and Training Director of the doctoral program in school psychology at the University of Massachusetts Amherst. Her research interests are focused on supporting mental health in learning environments, culturally responsive practices, and consultation. She currently serves as Co-Director of the Behavioral Health Integrated Resources for Children (BIRCH) Project, funded by Boston Children's Hospital.

Alexandra A. Lauterbach, Ph.D., is an associate professor of special education at the University of Massachusetts Amherst. Her scholarly interest centers on effective literacy instruction for students with high incidence disabilities, and effective professional development for their teachers. Dr. Lauterbach specializes in the use of qualitative methodology, and has served as a methodologist in special education, education leadership, higher education, school counseling, and school psychology.

Manuscript received December 31, 2020 Final revision received March 20, 29, 2021

Accepted March 30, 2021 